Pacific Journal of Mathematic 


\title{
THEOREMS ON GENERALIZED DEDEKIND SUMS
}

\author{
T. M. Apostol
}

1. Introduction. Generalized Dedekind sums $s_{p}(h, k)$, defined by

$$
s_{p}(h, k)=\sum_{\mu=1}^{k-1} \frac{\mu}{k} \bar{B}_{p}\left(\frac{h \mu}{k}\right)=\sum_{\mu=1}^{k-1} \frac{\mu}{k} B_{p}\left(\frac{h \mu}{k}-\left[\frac{h \mu}{k}\right]\right),
$$

were introduced by the author [1]. The integers $h$ and $k$ are assumed relatively prime, $B_{p}(x)$ is the $p$-th Bernoulli function, $B_{p}(x)$ the $p$-th Bernoulli polynomial (for definitions see $[1 ;(2.11),(2.12)]$ ), and $[x]$ is the greatest integer $\leq x$. For even values of the integer $p$ the sums $(1)$ are trivial $($ see $[1 ;(4.13)])$ and we assume in what follows that $p$ is odd. These sums enjoy a reciprocity law, namely;

$$
\begin{aligned}
(p+1)\left(h k^{p}\right. & \left.s_{p}(h, k)+k h^{p} s_{p}(k, h)\right) \\
& =p B_{p+1}+\sum_{s=0}^{p+1}\left(\begin{array}{c}
p+1 \\
s
\end{array}\right)(-1)^{s} B_{s} B_{p+1-s} h^{s} k^{p+1-s} .
\end{aligned}
$$

The $B$ 's being Bernoulli numbers*. An arithmetic proof of (2) is given in [1] by a method closely related to a general summation technique recently developed by Mordell [5]. When $p=1$, the sums

$$
s_{1}(h, k)=\sum_{\mu=1}^{k-1} \frac{\mu}{k}\left(\frac{h \mu}{k}-\left[\frac{h \mu}{k}\right]-\frac{1}{2}\right)
$$

are known as Dedekind sums and are usually denoted by $s(h, k)$. Aside from being of interest from an arithmetical standpoint, these sums also occur in the asymptotic theory of partitions and have been studied in a large number of papers, for example [1], [3], [5], [6], [7], [8], [9], [10], and [11].

In this paper we establish a connection between the sums (1) and certain finite sums involving Hurwitz zeta functions which makes it possible to give a short analytic proof of (2).

* When $p>1$, the factor $(-1)^{s}$ may be suppressed in the summand in (2) because the terms corresponding to odd values of $s$ vanish.

Received September 11, 1951.

Pacific J. Math. 2 (1952), 1-9 
2. The Hurwitz zeta function and Dedekind sums. The Hurwitz zeta function $\zeta(s, a)$ is defined for $\Re(s)>1$ and $a \neq 0,-1,-2, \ldots$, by the series

$$
\zeta(s, a)=\sum_{n=0}^{\infty}(n+a)^{-s}
$$

and its relation to $s_{p}(h, k)$ is given in the following theorem.

THEOREM 1. For odd $p>1$ we have

$$
s_{p}(h, k)=i p !(2 \pi i k)^{-p} \sum_{\mu=1}^{k-1} \cot \frac{\pi h \mu}{k} \zeta\left(p, \frac{\mu}{k}\right)
$$

while for $p=1$ we have the two equivalent expressions

$$
s(h, k)=\frac{1}{4 k} \sum_{\mu=1}^{k-1} \cot \frac{\pi h \mu}{k} \cot \frac{\pi \mu}{k}
$$

and

$$
s(h, k)=\frac{-1}{2 \pi k} \sum_{\mu=1}^{k-1} \cot \frac{\pi h \mu}{k} \frac{\Gamma^{\prime}(\mu / k)}{\Gamma(\mu / k)}
$$

Formula (5) is due to Rademacher [8], who derived it from the Fourier series expansion of (3). We will give here a purely arithmetic proof of (5) based on finite rather than infinite Fourier series. Secondly, we establish the equivalence of (5) and (6) and then prove (4). Finally, we indicate how (5) and (6) can be thought of as limiting cases of (4).

Proof of (5): The function $\bar{B}_{1}(x)$ is given by

$$
\bar{B}_{1}(x)= \begin{cases}x-[x]-1 / 2 & \text { if } x \neq \text { integer } \\ 0 & \text { otherwise. }\end{cases}
$$

Therefore, by formula (2.5) of [10] we may write

$$
s(h, k)=\sum_{\mu \bmod k} \bar{B}_{1}(\mu / k) \bar{B}_{1}(h \mu / k) .
$$

From Eisenstein's finite Fourier series expansion $[4 ;$ p. 318] we have 
(8)

$$
\bar{B}_{1}(h \mu / k)=-\frac{1}{2 k} \sum_{\nu=1}^{k-1} \sin \frac{2 \pi h \nu \mu}{k} \cot \frac{\pi \nu}{k}
$$

Using (8) in each factor of the summand in (7), we obtain

(9) $s(h, k)=\frac{1}{8 k^{2}} \sum_{\lambda=1}^{k-1} \sum_{\nu=1}^{k-1} \cot \frac{\pi \nu}{k} \cot \frac{\pi \lambda}{k} \sum_{\mu \bmod k}\left(\cos \frac{2 \pi \mu(\lambda-\nu h)}{k}\right.$

$$
\left.-\cos \frac{2 \pi \mu(\lambda+\nu h)}{k}\right)
$$

because of the identity $2 \sin x \sin y=\cos (x-y)-\cos (x+y)$. Since we have

$$
\sum_{\mu \bmod k} \cos \frac{2 \pi \mu(\lambda \pm \nu h)}{k}= \begin{cases}k & \text { if } \lambda \pm \nu h \equiv 0(\bmod k), \\ 0 & \text { otherwise }\end{cases}
$$

for each fixed $\nu$ only one value of $\lambda$ gives a nonzero contribution to each sum in the second member of (9), namely $\lambda \equiv \nu h(\bmod k)$ in the first sum and $\lambda \equiv-\nu h$ $(\bmod k)$ in the second. Therefore we have

$$
s(h, k)=\frac{1}{8 k} \sum_{\nu=1}^{k-1} \cot \frac{\pi \nu}{k} \cot \frac{\pi h \nu}{k}-\frac{1}{8 k} \sum_{\nu=1}^{k-1} \cot \frac{\pi \nu}{k} \cot \frac{-\pi h \nu}{k},
$$

and this is the same as (5).

Proof that (5) and (6) are equivalent: The relation [2; p. 163]

$$
\frac{\Gamma^{\prime}(\mu / k)}{\Gamma(\mu / k)}=-\gamma-\log k-\frac{\pi}{2} \cot \frac{\pi \mu}{k}
$$

$$
+\sum_{n \leq k / 2}^{\prime} \cos \frac{2 \pi n \mu}{k} \log \left(2-2 \cos \frac{2 \pi n}{k}\right),
$$

where $\gamma$ is Euler's constant and the prime indicates that when $k$ is even the last term is to be multiplied by $1 / 2$, is due to Gauss. Multiplying both sides of (10) by cot $(\pi h \mu / k)$ and summing on $\mu$ shows the equivalence of (5) and (6) upon observing that we have 


$$
\sum_{\mu=1}^{k-1} f(\mu)=0
$$

whenever $f$ is an odd function of $\mu$ which is also periodic mod $k$.

Proof of (4): Formula (4.11) of [1] gives a representation of $s_{p}(h, k)$ as an infinite series which, with some simplification, can be written in the form

$$
s_{p}(h, k)=i p !(2 \pi i)^{-p} \sum_{\substack{n=1 \\ n \neq 0(\bmod k)}}^{\infty} n^{-p} \cot \frac{n \pi h}{k} .
$$

Writing $n=q k+\mu$, with $q=0,1,2, \ldots, \infty$, and $\mu=1,2, \cdots, k-1$, we obtain

$$
\begin{aligned}
s_{p}(h, k) & =i p !(2 \pi i)^{-p} \sum_{\mu=1}^{k-1} \sum_{q=0}^{\infty}(q k+\mu)^{-p} \cot \frac{\pi h \mu}{k} \\
& =i p !(2 \pi i k)^{-p} \sum_{\mu=1}^{k-1} \cot \frac{\pi h \mu}{k} \zeta(p, \mu / k),
\end{aligned}
$$

where we must assume $p>1$ in order to insure that the series involved should be absolutely convergent and the rearrangements valid. This proves (4). We cannot hope for a proof of (4) along the lines of our proof of (5) because of the nonelementary nature of $\zeta(s, a)$.

If in (4) we replace $p$ ! by $\Gamma(p+1)$ and let $p$ be a complex variable which tends to 1 , then we can show that the two expressions for $s(h, k)$ in (5) and (6) occur naturally as limiting cases of the right member of (4). We first observe that, although the function $\zeta(s, a)$ has a pole at $s=1$, the sum

$$
\sum_{\mu=1}^{k-1} \cot \frac{\pi h \mu}{k} \zeta(s, \mu / k)
$$

is regular at $s=1$. This is easily seen by using the expansion

$$
\zeta(s, a)=\frac{1}{s-1}-\frac{\Gamma^{\prime}(a)}{\Gamma(a)}+\mathrm{O}(s-1)
$$

obtained from Whittaker and Watson [12; p. 271], substituting in (12) and using (11) to obtain 


$$
\lim _{s \rightarrow 1} \sum_{\mu=1}^{k-1} \cot \frac{\pi h \mu}{k} \zeta(s, \mu / k)=-\sum_{\mu=1}^{k-1} \cot \frac{\pi h \mu}{k} \frac{\Gamma^{\prime}(\mu / k)}{\Gamma(\mu / k)},
$$

which shows that the right member of (4) tends to the right member of (6) as $p \rightarrow 1$.

The connection between (5) and (4) can be obtained by using Hurwitz's functional equation in the form given by Rademacher [6; (1.24)], namely:

$$
\begin{aligned}
\zeta(s, \mu / k)=2 \Gamma(1-s)(2 \pi k)^{s-1} & \sum_{\lambda=1}^{k}\left(\cos \frac{\pi s}{2} \sin \frac{2 \pi \lambda \mu}{k}\right. \\
& \left.+\sin \frac{\pi s}{2} \cos \frac{2 \pi \lambda \mu}{k}\right) \zeta\left(1-s, \frac{\lambda}{k}\right),
\end{aligned}
$$

this being valid for $s=1,1 \leq \mu \leq k$. Multiplying by $\cot (\pi h \mu / k)$, summing on $\mu$ and using (11) leads to

$$
\sum_{\mu=1}^{k-1} \cot \frac{\pi h \mu}{k} \zeta(s, \mu / k)
$$

(13)

$$
=2 \Gamma(1-s)(2 \pi k)^{s-1} \cos \frac{\pi s}{2} \sum_{\lambda, \mu=1}^{k-1} \cot \frac{\pi h \mu}{k} \sin \frac{2 \pi \lambda \mu}{k} \zeta(1-s, \lambda / k) .
$$

Since $\zeta(0, a)=1 / 2-a$, when $s$ tends to 1 the right member of (13) approaches the value

$$
\begin{aligned}
\frac{1}{2 k} \sum_{\lambda=1}^{k-1} \sum_{\mu=1}^{k-1} \cot \frac{\pi h \mu}{k} \sin & \frac{2 \pi \lambda \mu}{k}\left(\frac{1}{2}-\frac{\lambda}{k}\right) \\
& =\frac{-1}{2 k^{2}} \sum_{\mu=1}^{k-1} \cot \frac{\pi h \mu}{k} \sum_{\lambda=1}^{k-1} \lambda \sin \frac{2 \pi \lambda \mu}{k}
\end{aligned}
$$

because of (11). Noticing that the last sum on $\lambda$ is the imaginary part of the sum

$$
\sum_{\lambda=1}^{k-1} \lambda e^{2 \pi i \lambda \mu / k}=k /\left(e^{2 \pi i \mu / k}-1\right)=-\frac{k i}{2} \cot (\pi \mu / k)-\frac{k}{2},
$$


we see that the right member of (4) also tends to the right member of (5) when $p \rightarrow 1$.

3. Proof of the reciprocity law. We can now give a proof of the reciprocity law (2) using complex integration. This proof is of additional interest in that we use properties of $\zeta(s, a)$ for fixed $s$ and variable $a$. We will need the following facts about $\zeta(s, a)$ :

$$
\begin{aligned}
& \zeta(s, a)=\zeta(s, a+1)+a^{-s}, \\
& \text { (15) } \quad \zeta(s, a+1)=\zeta(s)-s \zeta(s+1) a+O\left(a^{2}\right) \\
& \text { (16) } \quad \text { for } 0 \leq \Re(a) \leq M,(M \text { fixed }), \zeta(s, a) \text { tends uniformly to } 0 \text { as } \Im(a) \rightarrow 0, \\
& \pm \infty .(\text { The uniformity is with respect to } \Re(a)) .
\end{aligned}
$$

Equation (14) follows at once from the definition of $\zeta(s, a)$ and (15) is merely the beginning of the Taylor series for $\zeta(s, a+1)$ near $a=0$. Here $\zeta(s)=$ $\zeta(s, 1)$ is Riemann's zeta function. Relation (16) can be readily obtained, for example, by applying the Riemann-Lebesgue theorem to the integral representation [2; p. 266] :

$$
\Gamma(s) \zeta(s, a)=\int_{0}^{\infty} \frac{t^{s-1} e^{-t \Re(a)}}{1-e^{-t}} e^{-i t \Im(a)} d t,
$$

valid for $\Re(s)>1$ and $\Re(a)>0$. This gives (16) for $0<\Re(a) \leq M$ and (14) proves it for $0 \leq \Re(a) \leq M$.

Because of (4), the reciprocity formula (2) can now be put into the following form:

THEOREM 2. For odd $p>1$ we have

$$
\frac{i(p+1) !}{(2 \pi i)^{p}}\left\{h \sum_{\mu=1}^{k-1} \cot \frac{\pi h \mu}{k} \zeta(p, \mu / k)+k \sum_{\nu=1}^{h-1} \cot \frac{\pi k \nu}{h} \zeta(p, \nu / h)\right\}
$$

$$
=p B_{p+1}+\sum_{s=0}^{p+1}\left(\begin{array}{c}
p+1 \\
s
\end{array}\right) B_{s} B_{p+1-s} h^{s} k^{p+1-s} .
$$

Proof. We apply Cauchy's residue theorem to the function

$$
f(z)=\cot \pi z \cot (\pi h z / k) \zeta(p, z / k),
$$


Integrating in the positive sense around a contour $C$ consisting of a rectangle whose vertices are the points $\pm i T, k \pm i T$, with small semi-circular detours $C_{0}$ and $C_{k}$ around the points $z=0, z=k$, traversed along the $\operatorname{arcs} z=\epsilon e^{i \theta}$ and $z=k+\epsilon e^{i \theta}$, respectively, where $\pi / 2 \leq \theta \leq 3 \pi / 2$, and $0<\epsilon<1 / h$. Ultimately, $\epsilon$ will tend to 0 and $T>1 / 2$ will tend to $\infty$. The integrand $f(z)$ has first order poles at the points $z=1,2, \ldots, k-1$ due to the factor cot $\pi z$, and at the points $z=k / h, 2 k / h, \cdots,(h-1) k / h$ because of the factor $\cot (\pi h z / k)$. By (14) we have

$$
\zeta(p, z / k)=\zeta(p, z / k+1)+(k / z)^{p},
$$

so that the point $z=0$ is a pole of order $p+2$ for $f(z)$. Using the power series expansion

$$
\pi z \cot \pi z=\sum_{n=0}^{\infty} \frac{(2 \pi i)^{n} B_{n}}{n !} z^{n}
$$

in the neighborhood of $z=0$ (with the understanding that $B_{1}$ should be replaced by 0 ), and (15) with $a=z / k$ we find that Cauchy's theorem gives us

$$
\begin{aligned}
\frac{1}{2 \pi i} \int_{C} f(z) d z & =\frac{1}{\pi} \sum_{\mu=1}^{k-1} \cot \frac{\pi h \mu}{k} \zeta(p, \mu / k) \\
& +\frac{k}{\pi h} \sum_{\nu=1}^{h-1} \cot \frac{\pi k \nu}{h} \zeta(p, \nu / h)-\frac{p}{\pi^{2} h} \zeta(p+1)+R_{0},
\end{aligned}
$$

where

$$
R_{0}=\operatorname{Res}_{z=0} \cot \pi z \cot (\pi h z / k)(k / z)^{p} .
$$

We now observe that by periodicity of the cotangent and by (14), the contribution to the integral from the part of $C$ consisting of vertical line segments is

$$
\left(\int_{i T}^{i \epsilon}+\mathcal{f}_{-i \epsilon}^{-i T}\right) \cot \pi z \cot (\pi h z / k)(k / z)^{p} d z,
$$

and this vanishes since the integrand is an odd function of $z$. Next, the integrals along the horizontal segments tend to zero as $T \rightarrow \infty$ since, for $0 \leq x \leq k$ we have cot $\pi(x+i y) \rightarrow \mp i$ and, by (16), $\zeta(p,(x+i y) / k)$ tends to 0 uniformly 
in $x$ as $y \rightarrow \pm \infty$. Finally, combining the integrals over $C_{0}$ and $C_{k}$ by means of (14) and letting $T \rightarrow \infty$ we obtain

$$
\lim _{T \rightarrow \infty} \int_{C} f(z) d z=\int_{C_{0}} \cot \pi z \cot (\pi h z / k)(k / z)^{p} d z
$$

When $\epsilon \rightarrow 0$ we find

$$
\lim _{\epsilon \rightarrow 0} \int_{C_{0}}=\pi i R_{0}
$$

so that equation (19) leads to the result

(20)

$$
\frac{1}{\pi} \sum_{\mu=1}^{k-1} \cot \frac{\pi h \mu}{k} \zeta(p, \mu / k)+\frac{k}{\pi h} \sum_{\nu=1}^{h-1} \cot \frac{\pi k \nu}{h} \zeta(p, \nu / h)
$$

$$
=\frac{p}{\pi^{2} h} \zeta(p+1)-\frac{1}{2} R_{0}
$$

From (18) we easily calculate that

$$
R_{0}=\frac{2 i(2 \pi i)^{p}}{\pi h(p+1) !} \sum_{s=0}^{p+1}\left(\begin{array}{c}
p+1 \\
s
\end{array}\right) B_{s} B_{p+1-s} h^{s} k^{p+1-s},
$$

and, since we have

$$
\zeta(p+1)=-\frac{(2 \pi i)^{p+1} B_{p+1}}{2(p+1) !}
$$

equation (20) yields (17) and the proof is complete.

In [8], Rademacher gives a proof for the case $p=1$ using (5) instead of (4). Apparently unaware of [8], K. Iseki [3] has given a proof very much like Rademacher's analytic proof for the case $p=1$ in a recent paper.

\section{REFERENCES}

1. T. M. Apostol, Generalized Dedekind sums and transformation formulae of certain Lambert series, Duke Math. J., 17 (1950), 147-157.

2. G. Brunel, Bestimmte Integrale, Encyklopädie der Mathematischen Wissenschaften, II A 3, 135- 188 .

3. Kanesiroo Iseki, Analytic proof for the reciprocity law of Dedekind sums, Sugaku, 2, no. 3 (1950), 240-241. (Japanese) 
4. G. B. Mathews, Theory of Numbers, Cambridge, 1892.

5. L. J. Mordell, The reciprocity formula for Dedekind sums, Amer. J. Math., 73 (1951), 593-598.

6. Hans Rademacher, Zur Theorie der Modulfunktionen, J. Reine Angew. Math. 167 (1932), $312-336$.

7. Eine arithmetische Summenformel, Monatsh. Math. Phys., 39 (1932), $221-228$.

8. Egy reciprocitásképletröl a modülfuggvények elméletéböl, Mat. Fiz. Lapok, 40 (1933), 24-34.

9. - Die Reziprozitätsformel für Dedekindsche Summen, Acta Univ. Szeged. Sect. Sci. Math., 12, B (1950), 57-60.

10. - and Albert Whiteman, Theorems on Dedekind sums, Amer. J. Math., 63 (1941), $377-407$.

11. L. Rédei, Elementarer Beweis und Verallgemeinerung einer Reziprozitätsformel von Dedekind, Acta Univ. Szeged. Sect. Sci. Math., 12, B (1950), 236-239.

12. E. T. Whittaker and G. N. Watson, Modern Analysis, University Press, Cambridge, 1945.

California Institute of Technology 


\section{EDITORS}

HERBERT BUSEMANN

University of Southern California

Los Angeles 7, California
R. M. ROBINSON

University of California

Berkeley 4, California

E. F. BECKENBACH, Managing Editor

University of California

Los Angeles 24, California

\section{ASSOCIATE EDITORS}

\author{
R. P. DILWORTH \\ HERBERT FEDERER \\ MARSHALL HALL
}

P. R. HALMOS

HEINZ HOPF

R. D. JAMES

\author{
BØRGE JESSEN \\ PAUL LÉVY \\ GEORGE PÓLYA
}

J. J. STOKER
E. G. STRAUS

KÖSAKU YOSIDA

\section{SPONSORS}

UNIVERSITY OF BRITISH COLUMBIA

CALIFORNLA INSTITUTE OF TECHNOLOGY

UNIVERSITY OF CALIFORNIA, BERKELLEY

UNIVERSITY OF CALIFORNIA, DAVIS

UNIVERSITY OF CALIFORNIA, LOS ANGELES

UNIVERSTTY OF CALIFORNIA, SANTA BARBARA

OREGON STATE COLLEGE

UNIVERSITY OF OREGON
UNIVERSETY OF SOUTHERN CALIFORNIA STANFORD UNIVERSITY

WASHINGTON STATE COLLEGE UNIVERSTTY OF WASHINGTON

AMERICAN MATHEMATICAL SOCIETY NATIONAL BUREAU OF STANDARDS, INSTITUTE FOR NUMERICAL ANALYSIS

Vari-Type Composition by

Elaine Barth

Delores Gilbertson

With the cooperation of

E. F. Beckenbach

E. G. Straus

Printed in the United States of America by

Edwards Brothers, Inc., Ann Arbor, Michigan

UNIVERSITY OF CALIFORNIA PRESS * BERKELEY AND LOS ANGELES

COPYRIGHT 1952 BY PACIFIC JOURNAL OF MATHEMATICS 


\section{Pacific Journal of Mathematics}

\section{Vol. 2, No. $1 \quad$ January, 1952}

Tom M. (Mike) Apostol, Theorems on generalized Dedekind sums ........ 1

Tom M. (Mike) Apostol, Addendum to 'On the Lerch zeta function' ........ 10

Richard Arens, Extension of functions on fully normal spaces ........... 11

John E. Maxfield, A short proof of Pillai's theorem on normal numbers.... 23

Charles B. Morrey, Quasi-convexity and the lower semicontinuity of multiple integrals................................ 25

P. M. Pu, Some inequalities in certain nonorientable Riemannian manifolds....................................... 55

Paul V. Reichelderfer, On the barycentric homomorphism in a singular

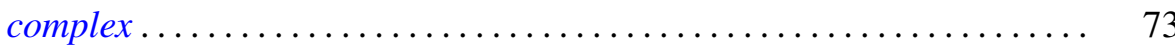

A. H. Stone, Incidence relations in multicoherent spaces. III ........... 99 